\title{
Linear and Branched Lactide Polymers for Targeted Drug Delivery Systems
}

\author{
V. I. Gomzyak ${ }^{a, *}$, N. G. Sedush ${ }^{a}$, A. A. Puchkov ${ }^{a}$, D. K. Polyakov ${ }^{a}$, and S. N. Chvalun ${ }^{a}$ \\ ${ }^{a}$ National Research Center Kurchatov Institute, Moscow, 123182 Russia \\ *e-mail:vgomzyak@gmail.com
}

Received December 15, 2020; revised January 30, 2021; accepted February 12, 2021

\begin{abstract}
The review presents modern advances in the synthesis of biodegradable polymers based on lactide of various topologies and also analyzes the main methods for preparation of nanoparticles that show promise for the creation of targeted drug delivery systems.
\end{abstract}

DOI: $10.1134 / \mathrm{S} 1560090421030064$

\section{INTRODUCTION}

Polymeric nanoparticles and microparticles are a promising platform for the development of targeted and prolonged-release therapeutic systems. Nanoformulations are characterized by increased efficacy and reduced side effects in comparison with classical dosage forms. Most of these systems are based on biodegradable and biocompatible materials, mainly aliphatic polyesters and polyethers, polyanhydrides, polyamides, and polyorthoesters. The most demanded of these are polyesters of $\alpha$-hydroxyacids: polylactide, polyglycolide, poly( $\varepsilon$-caprolactone), polydioxanone, and their copolymers [1-6]. The regulation of the molecular and supramolecular structure as well as the architecture of biodegradable polymers provides a way to control their physicochemical properties and the release profile of the active substance, which is important for the creation of prolongedrelease pharmaceutical systems. Currently, the literature describes a huge number of lactide polymers of different topology: linear, branched, star-shaped, and graft. This review summarizes the published data on methods for the synthesis of biodegradable polymers based on lactide of various topologies as well as on the main technologies for formulation of nanostructures for use in targeted drug delivery systems.

\section{COPOLYMERS OF LACTIDE AND GLYCOLIDE}

In recent decades, ring-opening polymerization (ROP) has attracted increased attention owing to the possibility of synthesis a wide range of biodegradable polymer structures compatible with the human body. In the body, the process of hydrolytic degradation (breaking of ester bonds) of these polymers proceeds, which at a later stage is supplemented by biodegradation under the action of enzymes. The products of hydrolysis are included in metabolism, and the rate of hydrolysis can be easily regulated by changing the chemical composition and structure of the copolymer $[2,4]$.

Some of the ROP polymerizable cyclic monomers are presented below.<smiles>CC1OC(=O)C(C)OC1=O</smiles>

Lactide<smiles>O=C1COC(=O)CO1</smiles>

Glycolide<smiles>O=C1COCCO1</smiles>

para-Dioxanone<smiles>O=C1CCCCCO1</smiles>

$\varepsilon$-Caprolactone<smiles>O=C1OCCCO1</smiles>

Trimethylene carbonate
Among them, lactide and its copolymers attract the most attention as materials for biodegradable surgical sutures, drug delivery systems, cardiovascular, dental and orthopedic temporary fasteners, and matrices for tissue engineering and bioartificial organs The main raw material for the production of poly(lactic acid) as well as the internal cyclic diester of lactic acid (lactide) is lactic acid. Lactic acid ( $\alpha$-hydroxypropionic acid) is 
the simplest $\alpha$-hydroxy acid with an asymmetric carbon atom. It exists in three forms: two optically active forms (enantiomers) and an optically inactive racemic mixture from which optically active $L$ - and $D$-isomers of lactide and optically inactive meso- and $D, L$-forms are produced [7-11]:<smiles>C[C@@H]1OC(=O)[C@@H](C)OC1=O</smiles>

$L, L$-Lactide $\left(T_{\mathrm{m}}=95-98^{\circ} \mathrm{C}\right)$<smiles>C[C@@H]1OC(=O)[C@@H](C)OC1=O</smiles>

meso-Lactide $\left(T_{\mathrm{m}}=52^{\circ} \mathrm{C}\right)$<smiles>C[C@H](O)C(=O)O</smiles>

$D(R)$-Lactic acid $\left(T_{\mathrm{m}}=16.8^{\circ} \mathrm{C}\right)$
In industry, lactic acid is usually obtained from raw materials that contain glucose, sucrose, and maltose, for example, refined syrup, molasses, and starch (corn, potato). Lactic acid can also be obtained from petrochemical raw materials, but the enzymatic method is much more ecofriendly. Moreover, petrochemical synthesis usually produces an optically inactive mixture (about $50: 50$ ) of $L$ - and $D$-forms of lactic acid, while lactic acid produced by bacterial fermentation is formed almost exclusively in the $L$-form $[8,9]$.

There are two main methods for the synthesis of polylactide: the polycondensation of lactic acid and the ring-opening polymerization of lactide. In view of the need to remove the released water from the reaction medium in the polycondensation method, it is rather difficult to synthesize a polymer with a high molecular weight and good physical and mechanical characteristics which is suitable for the manufacture of medical devices. To shift the equilibrium and obtain a high molecular weight product, it is necessary to distill off or chemically bind water released in the polycondensation process. The molecular weight of poly(lactic acid) synthesized by the polycondensation method without the use of additional crosslinking agents, commonly, does not exceed $10 \times 10^{3}$.

The ROP of aliphatic cyclic esters is a constantly and dynamically developing area of research. The advantages of ROP over polycondensation are as follows: milder reaction conditions, the absence of byproducts, and the possibility to use even six- or seven-membered lactones $[4,6]$. The mechanisms of ionic and coordination polymerizations of aliphatic cyclic esters have been systematically considered since the late 1960s. These mechanisms provide a reasonably good control of polymer characteristics (i.e., predictable molecular weight, narrow molecular weight distribution, high conversion) and are especially well suited for macromolecular engineering to obtain homo- and copolymers of various architectures (e.g., binary and ternary block copolymers, graft, starshaped, hyperbranched) [4].

Depending on the structure of the monomer, the catalytic/initiating system, and the nature of the formed active centers, ROP can proceed via the free radical mechanism, the coordination-insertion mechanism, or the anionic (or cationic) mechanism [3]. Anionic and coordination ROP allow achieving the highest polymerization yields and molecular weights in a short reaction time. In the anionic ringopening polymerization (AROP), nucleophilic reagents (organometallic compounds, metal amides, amines, alkoxides, alcohols, water) are used as initiators. A huge number of ROP catalytic systems are known [4, 12].

Depending on the organometallic derivatives of metals (such as Al, Sn, Nd, Y, Yb, Sm, La, Fe, Zn, Zr, $\mathrm{Ca}, \mathrm{Ti}$, and $\mathrm{Mg}$ ), ROP proceeds mainly according to two key mechanisms. Some of them act as catalysts and activate the monomer by complexing with the carbonyl group. Then the polymerization is initiated by any nucleophile, for example, water or alcohol, which are present in the polymerization medium in the form of impurities or are added in a controlled amount. The organometallic compound plays the role of an initiator, and polymerization proceeds according to the coordination-insertion mechanism. In ROP, hydroxyl-containing initiator molecules can act as molecular weight regulators for polymers. The polymerization rate can be controlled using a wide range of catalytic systems, such as tin octanoate and 2-ethylhexanoic acid [4].

To obtain biodegradable materials with predetermined properties and degradation profiles, several forms of lactide are copolymerized with each other as 
well as copolymerized with other cyclic esters: glycolide, $\varepsilon$-caprolactone, $\delta$-valerolactone, trimethylene carbonate. The copolymers of lactide with glycolide (PLGA) are widely used in medicine. They are approved by the United States Food and Drug Administration (FDA). Like the corresponding homopolymers (PLA and PGA), PLGA are thermoplastic polyesters that are synthesized by ring-opening polymerization:<smiles>CC1OC(=O)C(C)OC1=O</smiles>

Lactide<smiles>CC(C)CC1COC(=O)CO1</smiles>

Glycolide

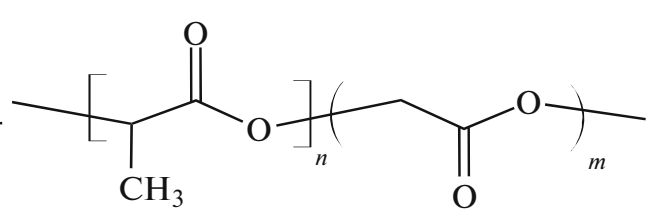

Poly(lactide-co-glycolide) (PLGA)
Owing to the different reactivity of lactide and glycolide, copolymers with different microstructures can be obtained. By varying the chemical composition, molecular weight, and supramolecular structure, one can "control" the physicomechanical characteristics of the material, its solubility, thermophysical properties, and the biodegradation rate. It is important to select the optimal reaction conditions to achieve high conversion and molecular weight of the polymer. The hydrophobicity of polylactide is much higher than that of polyglycolide owing to the presence of a pendant methyl group. As a result, the rate of hydrolysis of the main chain is much lower, while the solubility in organic solvents is higher.

Homopolymers of $L$-lactide and glycolide are highly crystalline materials with a relatively long degradation period, but their copolymerization disrupts the stereoregularity of chains and decreases the degree of crystallinity. Since the amorphous regions of the material are significantly more accessible for water than the crystalline ones, hydrolytic degradation in the copolymers of lactide with glycolide proceeds faster than that in the corresponding homopolymers. Copolymers containing more than $30 \%$ glycolide units are completely amorphous. The glass transition temperature varies from 40 to $60^{\circ} \mathrm{C}$ and depends on the microstructure of the copolymer and its composition. The rate of biodegradation also depends on the ratio of the units; copolymers with the composition lactide : glycolide $=50: 50$ degrade most rapidly. Owing to their biocompatibility as well as the ability to regulate the physical and mechanical properties and the time of biodegradation, lactide polymers and copolymers with glycolide are widely used for the manufacture of suture materials for surgery, fasteners for traumatology and orthopedics, matrices for regenerative medicine, and targeted delivery and prolonged drug release systems. Currently, commercially available PLGA copolymers of various compositions (50: $50,65: 35,75: 25$, and $85: 15)$ and molecular weight $\left((10-200) \times 10^{3}\right)$ are well studied. Most of the work is focused on the copolymers of two types: poly $(L-$ lactide-co-glycolide) (PLGA) and poly $(D, L$-lactide-coglycolide) (PDLGA). Miller et al. [13, 14] showed that
PDLGA of composition 50 : 50 degrades in about 12 months; PDLGA of composition $75: 25$ degrades in 4-5 months; PDLGA of composition $85: 15$ degrades in 5-6 months [15].

PLGA has low toxicity, excellent biological compatibility, and relatively high compatibility with other polymers and physiologically active substances; therefore, it is commonly used in medicine, in particular, to create drug-loaded nanoparticles and microparticles. A number of methods were developed that make it possible to prepare PLGA nanoparticles with a given size and degree of drug loading. The choice of method is mainly determined by the type of copolymer and drug and the method of administration to the body. Nano- and microparticles are formulated by chemical, physical, and physicochemical methods. Most of them are modifications of three main methods: spray drying, phase separation (coacervation), and solvent extraction (evaporation).

\section{Emulsion Methods}

Simple emulsions. The solvent extraction/evaporation method is the most common encapsulation method (Fig. 1). It does not require elevated temperatures or the introduction of additional components (e.g., phase separation agents). By careful selection of reagents and encapsulation conditions, nanoparticles and microparticles of controlled size can be obtained. The simplest version of this method involves the formation of oil-in-water or water-in-oil emulsions.

This method is commonly used for loading physiologically active substances insoluble or poorly soluble in water. PLGA is dissolved in a good, water-immiscible solvent (e.g., dichloromethane, chloroform, ethyl acetate) to give a single-phase solution. Because of its toxicity, chloroform is not recommended for use in the preparation of dosage forms. Dichloromethane is highly volatile and, as reported, uniformly spherical particles are formed with its use. Ethyl acetate is preferred because it is less toxic but partially miscible with water. Therefore, the search for a nontoxic solvent with appropriate properties is still an important issue for researchers using this method. A drug and polymer 


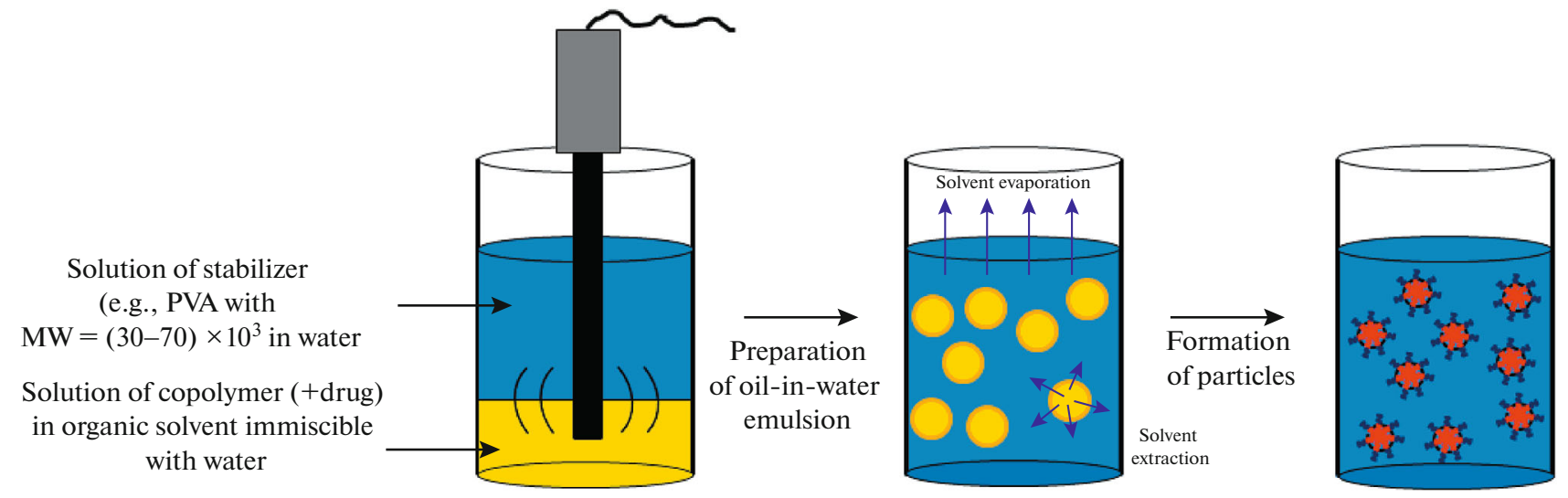

Fig. 1. Schematic illustrating the formation of nanoparticles by emulsifying an organic solution of a drug with a polymer in an aqueous medium followed by extraction/evaporation of the solvent. Color drawings can be viewed in the electronic version.

(e.g., PLGA) are dissolved in an organic solvent and then added to water that contains a surfactant (often poly(vinyl alcohol)) at the required temperature under stirring. Then, the organic solvent is gradually evaporated or extracted, and PLGA particles are precipitated in the form of spheres [16-19]. Initially, the emulsion is kept under reduced or atmospheric pressure, controlling the stirring rate while evaporating the solvent. Later, the emulsion is transferred to a large volume of water (with or without surfactant) or another quenching medium to remove the residual organic solvent. The prepared solid nanoparticles or microparticles are washed and dried under suitable conditions to obtain the final formulation for injection. By varying the stirring speed, surfactant concentration, and chemical structure of PLGA, the particle size can be tuned. To date, many hydrophobic drugs of different therapeutic groups were successfully loaded by this method: antagonists of narcotic analgesics (naltrexone, cyclazocine), local anesthetics (lidocaine), anticancer agents (cisplatin, 5-fluorouracil), steroids (progesterone), and many others [20]. The solvent evaporation method using an oil-in-water emulsion is suitable for the incorporation of hydrophobic compounds, but it is not effective for hydrophilic drugs. The loading of hydrophilic active compounds using an oil-in-water emulsion contributes to a low loading efficiency and sharp release profiles. In addition, the drug can diffuse into the continuous aqueous phase during emulsification, resulting in a large loss of the drug. To overcome these losses, several modified methods were proposed, among which one of the most effective is the method of double emulsion water in oil/in water.

Double emulsion method. Multiple emulsion techniques (e.g., water in oil/in water) are well suited for loading hydrophilic compounds and drugs, such as proteins, peptides, and hydrophilic anticancer agents. In contrast to simple emulsion methods, which are ideal for water-insoluble drugs, double emulsions of the drug to be incorporated into the particles are usually obtained as follows: an aqueous solution of a hydrophilic drug is prepared and emulsified in the organic phase which is a polymer solution in organic solvent. This results in the formation of a first (or primary) emulsion in which aqueous droplets containing the drug are suspended in the organic phase (water-inoil emulsion). The emulsion is then dispersed in a basic aqueous medium containing surfactants to prevent the aggregation of droplets, forming a double water/oil/water emulsion. The size of the resulting droplets can be controlled by changing the modes of dispersion and homogenization. The addition of the primary emulsion to the surfactant solution and the removal of the organic solvent lead to the formation of a rigid polymer shell around the hydrophilic drug substance. In this way, microparticles based on naltrexone hydrochloride, pseudoephedrine hydrochloride, leuprolide acetate peptide, vaccines, and other substances are obtained. PLGA-based particles are sensitive to moisture; therefore, they are dried by freezedrying for long-term storage, often by introducing cryoprotectants (usually sucrose or glucose) [21-23]. Compared to other methods of particle preparation, the solvent evaporation method is rather laborious. Its duration depends on the rate of evaporation of solvent, which can be increased by heating the system. Thus, the problem of drug stability arises especially when operating with temperature-sensitive substances. It is important to avoid residues of toxic solvents (methylene chloride, chloroform, acetonitrile, tetrahydrofuran, or methanol) in the final product, which is achieved through prolonged drying. Very often, the drying process has to be carried out at low temperatures owing to the low glass transition temperatures of the polymers used. It is reported that in some cases drying can take more than one week. The above disadvantages of the solvent evaporation method provide an argument for focusing on other microparticle preparation methods, such as spray drying, which can effec- 


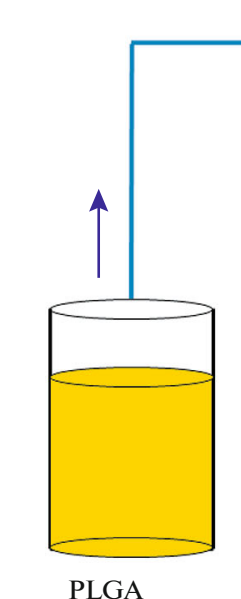

in organic solvent
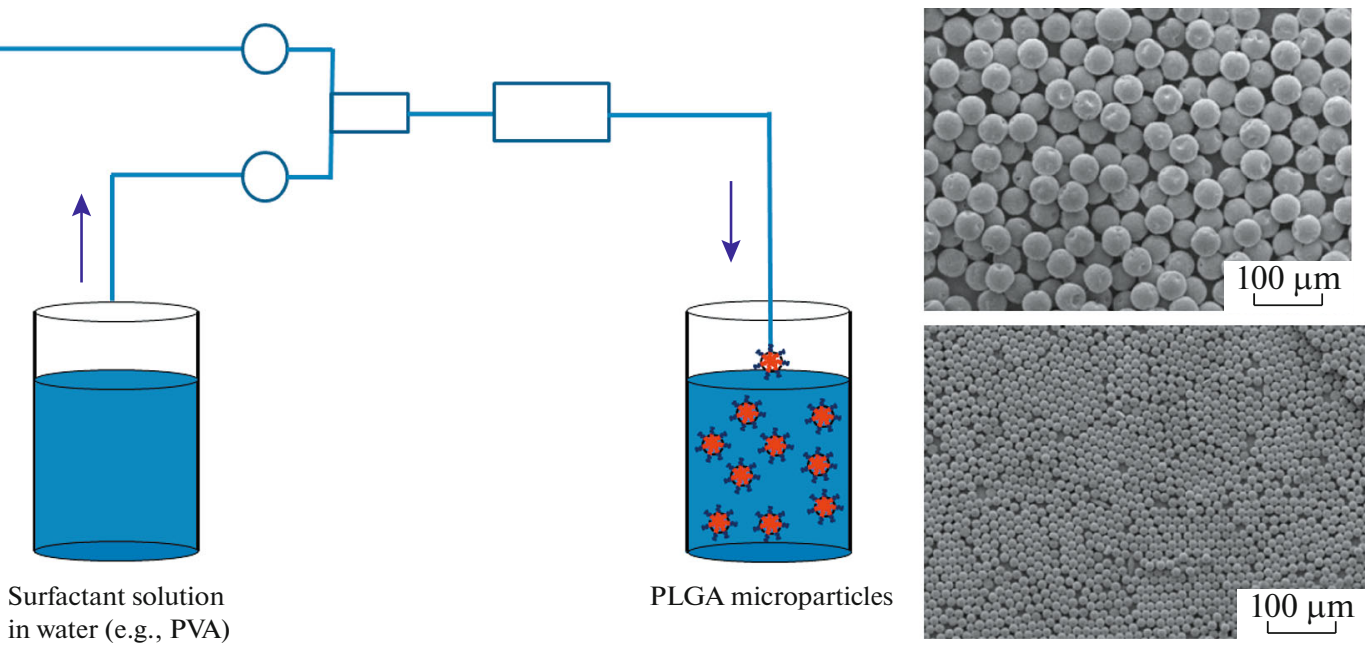

Fig. 2. Schematic illustrating the formation of PLGA nanoparticles by microfluidic technologies.

tively encapsulate a wide range of drugs, both hydrophobic and hydrophilic, with shorter preparation times and milder drying conditions.

\section{Spray Drying}

Spray drying is a relatively simple, inexpensive, and effective method of producing polymer microparticles with an incorporated biologically active substance. The spray drying process is flexible and produces good quality microparticles. The type of drug (hydrophobic or hydrophilic) determines the choice of solvent that will be used in the process. Traditionally, the process consists of spraying a solution/solid dispersion of a polymer with an active substance into a hot dry medium, for example, into a stream of heated air. At the exit from the spray nozzle, the solution/dispersion stream is crushed into small droplets which, as a result of rapid evaporation of the solvent, transform into a solid dried state. This is done in three steps: aerosol formation, aerosol contact with warm air and aerosol drying, and separation of the dried product and solvent-saturated air. In this way, both microparticles with a relatively uniform distribution of the active substance over the volume (in this case, a joint solution of the polymer and the active substance in an organic solvent is subjected to spray drying) and microparticles of the core-shell type are obtained (in this case, the dispersion of the substance forming the core of a microparticle and insoluble in a dispersion medium, in the concentrated solution of a substance that forms a shell, is subjected to spray drying). The literature describes a technology for producing PLGA-based microspheres for the delivery of diazepam, piroxicam, progestone, theophylline, and vitamin $\mathrm{D}_{3}$ and for the encapsulation of water-soluble materials (albumin and vaccine antigens). The size of microsphere particles formulated by spray drying ranges from several microns to several tens of microns and has a relatively narrow distribution [24]. The spray drying of a PLGA solution with an active substance is often carried out in the presence of an aqueous solution of carbohydrates (mannitol, lactose, trehalose); in addition, stabilizers (poly(ethylene glycols), polyethyleneimine) are added to reduce the tendency toward agglomeration.

\section{Microfluidic Technologies}

A promising approach for preparation of polymer particles is the use of microfluidic technologies (Fig. 2). This method implies the ability to manipulate fluid flows and single droplets, which in turn opens up wide opportunities for implementing microfluidic technologies for the synthesis and assembly of "smart" particles and the creation of microcapsules, polymerosomes, and biocompatible cell matrices as well as high-throughput methods of analysis in experimental biology. The generation of a monodisperse emulsion of controlled size and morphology under the mildest conditions is one of the main advantages of droplet microfluidics in comparison with classical approaches to emulsification based on mechanical action. This method allows one not only to fundamentally improve the quality of the obtained microparticles/nanoparticles but also to ensure the production of particles of complex structure (multilayer, multicore). The advantage of polymer particles obtained in microreactors is a narrower particle size distribution, which is achieved through the strict and complete control of conditions in the microreactor (pressure, temperature, and flow rate).

Microfluidic devices can be capillary systems with direct flows, focused flow, or a combination of both of these principles. In cocurrent capillary devices, the aqueous phase (e.g., PVA in water) is introduced into two side channels and the organic phase containing 
the drug and polymer is directed into the central channel of the device using syringe pumps at a constant speed. Monodisperse emulsion droplets are continuously formed at the junction points of the combined microchannels used in the capillary device with direct streams to prepare microspheres loaded with a drug (e.g., bupivacaine, a local anesthetic). In capillary flow focusing devices, two liquid phases (i.e., an organic phase containing drug and polymer and a continuous aqueous phase) are introduced from opposite directions into a microfluidic mixing cell. The internal organic phase is hydrodynamically focused by the flow of an external aqueous liquid through the opening. When the organic phase enters the hole, it decomposes (under conditions of dripping or insufficient blowing) to form droplets of the monodisperse emulsion [25-27]. Compared to the simple oil-in-water emulsion method, empty PLGA microspheres which were obtained by the flow focusing method had a narrower particle size distribution. All the methods used to produce microparticles can be employed to produce PLGA nanoparticles (nanospheres and nanocapsules) by adjusting the process parameters. Over the past two decades, many PLGA-based dosage forms have been developed and used to treat various diseases [28-30]. The most popular are PLGAs with a relative composition (molar ratio of lactide : glycolide) of $50: 50$ and $75: 25$ [31]. Owing to the hydrolysis of PLGA, properties such as the glass transition temperature $T_{\mathrm{g}}$, moisture content, and molecular weight change over time. These changes in properties of PLGA also affect the release rate of the loaded drug, which also depends on the nature of the drug [32].

Amphiphilic block copolymers based on lactide and ethylene oxide are a promising type of polymers for creating nanoparticles. Unlike PLGA particles, which are hydrophobic and require obligatory stabilization with surfactants, the tendency toward aggrega- tion of copolymers of lactide and ethylene oxide is even inherent in their very structure. The absence of the need to remove the free stabilizer provides a great technological advantage.

\section{BLOCK COPOLYMERS OF LACTIDE WITH POLY(ETHYLENE GLYCOL)}

Poly(ethylene glycol) (PEG) is a biocompatible, nontoxic, and nonimmunogenic water-soluble polymer widely used in pharmaceutical and biomedical technologies. Although PEG with molecular weight below a certain value does not degrade in biological media, it can be eliminated from the body through excretion. It was shown that $98 \%$ of PEG with a molecular weight of $4 \times 10^{3}$ is excreted from the human body [33]. The widespread use of PEG derivatives in targeted drug delivery systems is associated with PEG resistance to proteins, since the resulting hydrogen bonds of PEG with water exclude the adsorption of proteins on them. Since PEG is a readily soluble, highly polar polymer capable of forming a random coil, which can effectively sterically protect the surface of various nanoparticles and microparticles, block copolymers with various hydrophobic blocks can be obtained using PEG of different functionality and molecular weights. The ring-opening polymerization of lactide is a widely used method for the synthesis of binary and ternary block copolymers with PEG. Polymerization, usually, is carried out in bulk at $140-170^{\circ} \mathrm{C}$ in an inert medium using a catalyst and a macroinitiator (monofunctional methoxy(polyethylene glycol) for the synthesis of binary copolymers or bifunctional poly(ethylene glycol) for ternary block copolymers) [34]. ROP allows the synthesis of high molecular weight copolymers under mild conditions and the control of molecular weight and molecular weight distribution [4].

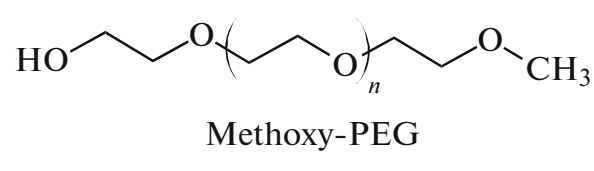

<smiles>CCOCCOCCO</smiles>

PEG

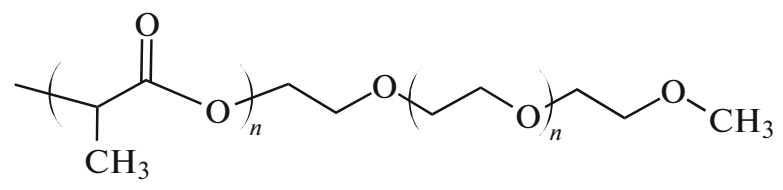

Binary block copolymer (PLA-MPEG)

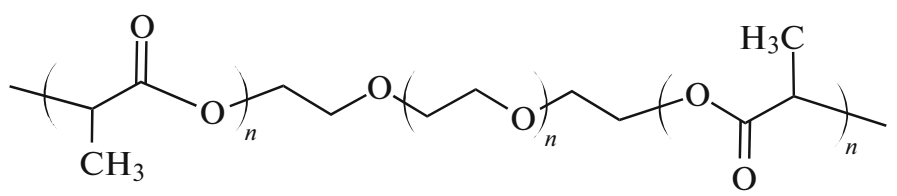

Ternary block copolymer (PLA-PEG-PLA) 
Table 1. Examples of star-shaped (3-, 4-, and 6-arm) PLA polymers synthesized by ROP (catalyst $\left.\mathrm{Sn}(\mathrm{Oct})_{2}\right)$

\begin{tabular}{l|c|c|c|c}
\hline \multicolumn{1}{c|}{ Core structure } & Arm structure & Number of PLA arms & $\begin{array}{c}\text { Molecular weight of } \\
\text { polymer } M_{\mathrm{n}} \times 10^{-3}\end{array}$ & References \\
\hline Glycerol & PDLLA & 3 & $4.0-100.0$ & {$[50]$} \\
Trimethylolpropane & PDLLA & 3 & $14.6-75.5$ & {$[50,51]$} \\
Pentaerythritol & PDLLA & 4 & $2.7-64.6$ & {$[50,52,53]$} \\
Dipentaerythritol & PDLLA & 6 & $5.9-86.0$ & {$[51,54,55]$} \\
Inositol & PDLLA & 6 & $4.6-66.8$ & {$[50,56]$} \\
Sorbitol & PDLLA & 6 & $1.0-33.6$ & {$[57,58]$} \\
Xylitol & PDLLA & 6 & $2.8-33.6$ & {$[57]$} \\
Cholic acid & PDLLA & 3 & $3.3-23.1$ & {$[50]$} \\
Trimethylolpropane & PLLA & 3 & $2.3-75.5$ & {$[59,60]$} \\
Glycerol & PLLA & 3 & $2.8-130.0$ & {$[61,62]$} \\
Pentaerythritol & PLLA & 4 & $2.7-320.0$ & {$[59,63,64,68]$} \\
Dipentaerythritol & PLLA & 6 & $2.3-88.0$ & {$[50,65,66]$} \\
Inositol & PLLA & 6 & $11.0-34.0$ & {$[50,56,67]$} \\
Sorbitol & PLLA & 6 & $1.0-37.0$ & {$[57,58]$} \\
Sorbitol & PLLA & 6 & $1.8-33.6$ & {$[57]$} \\
\hline
\end{tabular}

Such block copolymers can be modified at terminal hydroxyl groups. For example, Asadi et al. prepared PLA-PEG-PLA triblock copolymers with terminal diacrylic fragments [35]. They are also characterized by a good biocompatibility and the ability to self-associate in an aqueous medium, and the presence of terminal double bonds allows photocrosslinking of these micelles to obtain polymer nanogels. Organometallic compounds are commonly used to catalyze the ROP of lactide, and tin octanoate is one of the most popular catalysts for the polymerization of lactones in the presence of hydroxyl-containing compounds $[4,36]$. The purity of the polymer and its molecular mass distribution affect the expected key characteristics of nanostructures after aggregation (e.g., zeta potential, hydrodynamic particle diameter). In addition, the possible formation of a byproduct, a PLA homopolymer or free PEG blocks, can also affect the physicochemical characteristics of the resulting nanoparticles. For example, mixing the PLA homopolymer with PLA-PEG copolymers changes the surface density of PEG on PLA-PEG nanoparticles [37, 38]. As discussed above, the surface density of PEG is an important characteristic of nanoparticles that can affect colloidal stability as well as interactions with plasma proteins, including complement proteins. It is important to control the purity of the starting macroinitiators, since the presence of a PEG-diol impurity in methoxy-PEG used for the synthesis of diblock copolymers can lead to the formation of a triblock copolymer byproduct and, therefore, to a mixture of block copolymers of different topology [39].

The amphiphilic block copolymers of lactide with poly(ethylene glycol) in an aqueous medium are capable of self-assembly into supramolecular structures, micelles. In the simplest case, the formation of spherical micelles of the core-corona type occurs in which the hydrophobic PLA block forms the core and the hydrophilic PEG forms the corona. Micelles based on these block copolymers are biocompatible, biodegradable, and nontoxic, and the PEG forming the shell provides the necessary steric barrier and prevents the adsorption of plasma proteins on the surface of particles, thereby increasing the time of their circulation in the body. Thus, by varying the hydrophilic-lipophilic balance of amphiphilic copolymers as well as the stereospecificity and length of their constituent blocks, particles with controlled size and morphology can be obtained. Undoubtedly, such nanoparticles have great potential for development of prolonged and targeted drug delivery systems.

\section{STAR-SHAPED POLYLACTIDES}

The rapid development of synthetic methodologies allows to synthesize polymers with a complex archi- 
tecture (star-shaped, hyperbranched, dendritic, polymer brushes) in which polymer segments of different types (hydrophobic and/or hydrophilic, amorphous and/or crystalline) are combined in one macromolecule. Star-shaped polymers are the simplest branched polymers consisting of several linear chains connected to a central branching center. According to the IUPAC nomenclature, star-shaped polymers are characterized by the presence of a single branching point from which linear chains originate. The functionality of this point is indicated by the number of arms (linear chains) emanating from it. There are two main ways to synthesize star-shaped polymers: divergent ("core first") and convergent ("arm first"). Low molecular weight substances (e.g., polyatomic alcohols) or macromolecules with reactive functional groups are used as the core. The examples of initiators used for the synthesis of star-shaped polylactides are presented below.<smiles>OCC(O)CO</smiles>

Glycerol (3 arms)<smiles>OCC(O)C(O)CO</smiles>

Erythritol (4 arms)<smiles>OCC(CO)(CO)CO</smiles>

Pentaerythritol (4 arms)<smiles>OCC(O)C(O)C(O)CO</smiles>

Xylitol (5 arms)<smiles>OCC(O)C(O)C(O)C(O)CO</smiles>

Sorbitol (6 arms)<smiles>OCC(CO)(CO)COCC(CO)(CO)CO</smiles>

Dipentaerythritol (6 arms)
Star-shaped polymers have unique properties compared to their linear counterparts. Owing a high density of segments, branching leads to more compact structures, which sharply affects the mechanical and viscoelastic properties and characteristics of solutions [40]. The study of these properties is the subject of numerous theoretical and experimental studies. Starshaped polymers are synthesized using the "core first" and "arm first" strategies as well as their various combinations [41-49]. Table 1 shows the examples of starshaped polylactides synthesized in the presence of cores with different functionalities.

In the case of lactide derivatives, the core first strategy is used more widely than the arm first strategy. Star-shaped biodegradable polyesters are synthesized by the ROP method in bulk at $140-200^{\circ} \mathrm{C}$ in an inert atmosphere using a catalyst and a multifunctional initiator as well as in solvents, such as tetrahydrofuran, $m$ xylene, toluene, methylene chloride, chlorobenzene, chloroform, and DMSO. While linear polymers are synthesized by ROP with a monofunctional or difunctional alcohol, the use of a polyol results in highly branched (star-shaped) structures.

Typically, the number of "arms" in the polymer corresponds to the number of functional groups in the initiator. The position of the hydroxyl group in the core and the ratio of monomer to initiator determine whether all hydroxyl groups initiate the growth of the PLA chain. It was shown that primary hydroxyl groups are more effective in initiating lactide polymerization. The aim of study [68] was the systematic assessment of the effect of the amount of hydroxyl groups of the initiator on the polymerization rate and optimization of the polymerization time required for the formation of high molecular weight polylactides. The polymerization of lactide was carried out at $200^{\circ} \mathrm{C}$ (catalyst $\left.\mathrm{Sn}(\mathrm{Oct})_{2}\right)$, and the initiators used contained from 1 to 12 hydroxyl groups. It was shown that the rate of polymerization increases with an increase in the number of hydroxyl groups in the initiator, as is seen from the analysis of the kinetic curves. For all initiators, the lactide conversion was about $95 \%$. It follows from the results obtained that the functionality of the initiator affects the rate of polymerization, but not the monomer-polymer equilibrium. The use of initiators with different numbers of hydroxyl groups made it possible to obtain PLLA with different numbers of arms. An important condition for effective initiating activity is the solubility of the initiator in the polymerization mixture. A poor solubility is characteristic of initiators with a high melting point, such as pentaerythritol, dipentaerythritol, and $\beta$-cyclodextrin, which mark- 
edly complicates polymerization [69]. Using multifunctional initiators, various molecular architectures with a strictly defined number of arms from 3 to 32 can be obtained. For example, using dendritic initiators derived from 2,2-bis(hydroxymethyl)propionic acid (bis(MPA)), star-shaped PDLLA, PLLA, and PCL with 2, 4, 6, or 12 arms corresponding to the number of hydroxyls and a narrow MWD (less than 1.1) were obtained. Stars with a large number of arms (up to 160) are synthesized using hyperbranched polymers and/or dendrimers [44-48, 70, 71]. More complex polyols can also serve as initiators, for example, octafunctional polyhedral oligomeric silsesquioxane (POSS) [72]. In almost all works, $\mathrm{Sn}(\mathrm{Oct})_{2}$ was used as a catalyst. Other catalysts were also implemented such as calcium hydride, tin acetylacetonate, potassium hexamethyldisilazide, tetraphenyltin, $\mathrm{Bi}$ (III) acetate $\left(\mathrm{Bi}(\mathrm{OAc})_{3}\right)$, and even the enzyme lipase. The $\mathrm{Bi}(\mathrm{OAc})_{3}$-based system was comparable in catalytic efficiency to $\mathrm{Sn}(\mathrm{Oct})_{2}$ at $140^{\circ} \mathrm{C}$. There are few publications on the preparation of star-shaped polylactides by polycondensation. The synthesized polymers usually have relatively low molecular weights $\left(M_{\mathrm{n}}<4.7 \times\right.$ $\left.10^{3}\right)$. Higher molecular weight poly(lactic acid) $\left(M_{\mathrm{n}} \sim\right.$ $\left.67 \times 10^{3}\right)$ was obtained by the direct polycondensation of lactic acid initiated by dipentaerythritol and pentaerythritol with antimony trioxide and $p$-toluenesulfonic acid as catalysts, respectively. In addition, starshaped 3- and 4-arm PEG-PLA copolymers were obtained from branched poly(ethylene glycol) [7375]. Also, star-shaped polylactide was synthesized by the ROP of $D, L$-lactide in supercritical carbon dioxide with $D$-sorbitol as an initiator. This process was carried out at significantly lower temperatures (up to $80^{\circ} \mathrm{C}$ ) compared to bulk reactions $\left(140^{\circ} \mathrm{C}\right)$. As a result, a decrease in the formation of byproducts was observed, which led to a narrower polymer dispersion and good control of the degree of branching [76]. Usually, starshaped polylactides are synthesized by ROP; however, other approaches, for example, RAFT and ATRP, can be used [77, 78]. Many lactone-based star-shaped polymers can ultimately be used to produce nanoparticles for targeted drug delivery. Recently, it was shown that 3-, 4-, and 6-arm PLGA-PEG block copolymers (initiated by trimethylolpropane, pentaerythritol, and dipentaerythritol, respectively) exhibit reduced critical micelle concentrations, a slower release of encapsulated doxorubicin, and a certain increase in internalization into HeLa line cells [79]. These star-shaped polymers probably do not form monomolecular micelles: the average size of their aggregates in water is in the range of $80-200 \mathrm{~nm}$, and it increases with an increase in the degree of branching. This indicates that colloids are formed by polymer aggregation and become more stable because of more frequent entanglements as a result of the presence of branching points (hence the lower values of the critical micelle concentration). A number of studies demonstrated that nanoparticles based on star-shaped polymers can be loaded with drugs, such as indomethacin and ibuprofen [72, 80-82].

\section{BRANCHED POLYMERS BASED ON POLYGLYCEROL}

To obtain star-shaped polymers the number of arms in which is more than 10 , it is necessary to use initiators containing a large number of reactive groups, hyperbranched polymers and dendrimers. The physicochemical properties of hyperbranched polymers can be varied within a fairly wide range without significantly changing the initial macromolecule but only by varying the set of functional groups in terminal chains and the degree of their modification. In recent years, dendrimers as well as hyperbranched polymers have attracted serious attention owing to their unique properties related to their branched architecture and a large number of functional groups. A highly branched and dense but irregular structure of hyperbranched polymers leads to increased solubility in comparison with linear analogs, low solution viscosity, and high concentration of functional terminal groups [83-85].

The study of star-shaped polymers with a hyperbranched high molecular weight core is of particular interest. Hyperbranched polymers are a special type of dendritic polymer and have a very high branching density with the possibility of branching in each monomer unit. They are usually prepared in a onestep synthesis, which makes it difficult to control branching and molecular weight with high accuracy, resulting in products with a wide MWD. This distinguishes hyperbranched polymers from perfectly branched and monodisperse dendrimers. There are a large number of publications devoted to the study of hyperbranched polyglycerol and its derivatives in medical applications [81-89]. FDA-approved oligoglycerols and polyglycerols (PG) occupy a special position in the field of nanomedicine owing to their topological homogeneity, biocompatibility, and multifunctionality. In addition, PG can be degraded by enzymes to form nontoxic, easily excreted metabolites. For these reasons, PG found many uses as a drug delivery vehicle. PG is prepared by ring-opening polymerization. There are many approaches to the synthesis of PGs of different structures which assume a wide variety in the degree of branching, size, surface topology, and chemical properties in general. The synthesis of hyperbranched polyglycerol from glycidol is schematically shown below. 


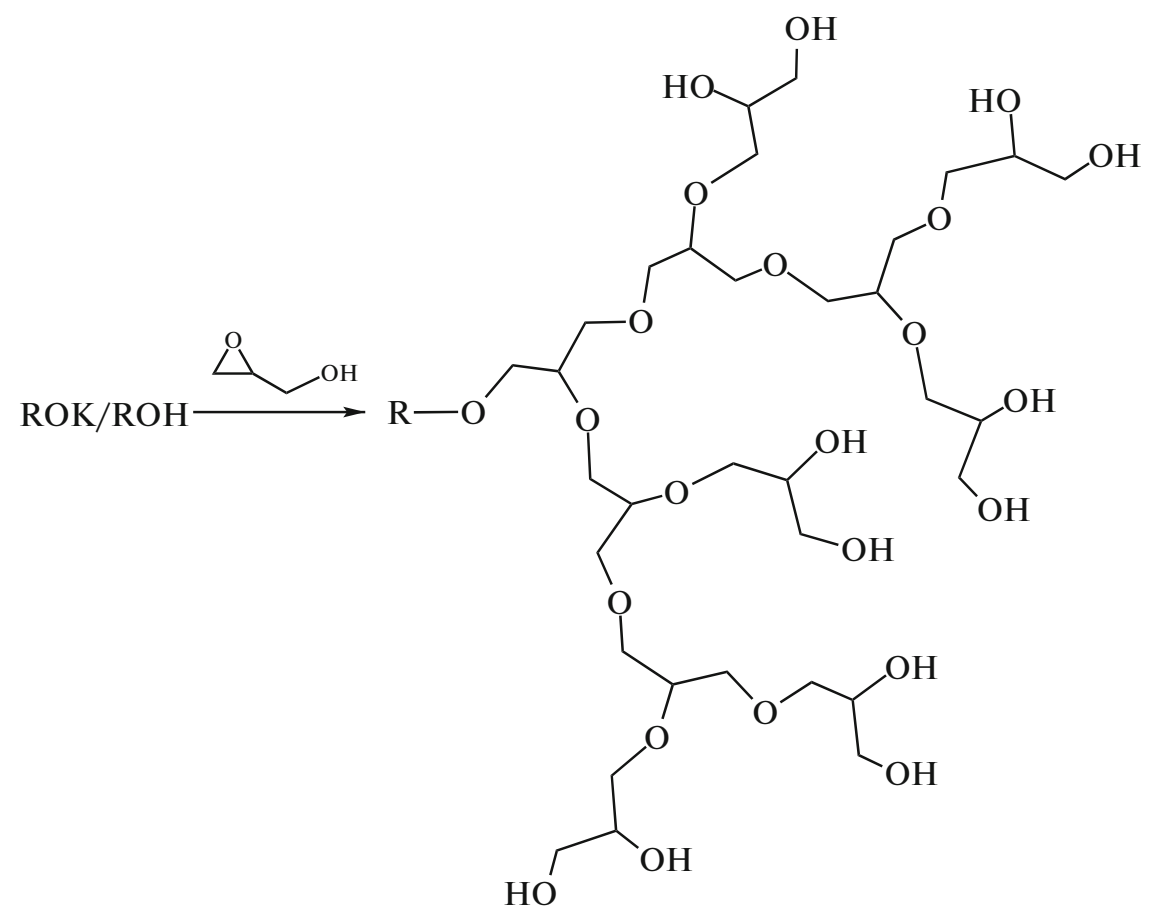

Along with the synthesis of hyperbranched polyglycerols (HBPG), in the last decade, pathways for the synthesis of ideal dendritic PGs, dendrons, microgels, and hydrogels were also reported. A high molecular weight PG is highly hydrophilic and has a large number of terminal hydroxyl groups which can be easily modified to obtain a wide range of compounds [86].

The modification of PG hydroxyl groups with hydrophobic blocks makes it possible to obtain amphiphilic systems with a hydrophilic core and a hydrophobic outer shell which exhibit host-guest properties. Over the past decades, a wide range of PGs of different topologies and molecular weights have been synthesized. Arvanitoyannis et al. [87] synthesized a threearm star-shaped PLLA via ROP using glycerol as a core. The synthesized polyester had a low degree of crystallinity, reduced melt viscosity, and high molecular weight. Multiarm star-shaped block copolymers were prepared using hyperbranched PG oligomers as initiators. At the first stage, a hyperbranched polyglycerol (core) was synthesized, and then the polymerization of $L$-lactide was carried out via the terminal groups of HBPG at various ratios; as a result, linear branched copolymers with different lengths of the polylactide block were formed [88]. The ROP of lactide was carried out at $115^{\circ} \mathrm{C}$ for $24 \mathrm{~h}$ (catalyst $\left.\mathrm{Sn}(\mathrm{Oct})_{2}\right)$. Similar linear dendritic copolymers can be obtained by the chemical bonding of polylactide blocks with HBPG by the carbodiimide method [89]. The advantage of this method was that all PLA branches had the same molecular weight. It was found that the release rate of quercetin (a poorly water-soluble drug) decreased with increase in $M_{\mathrm{n}}$ of copolymers HBPG-PLA. Copolymers with glycolide were synthesized similarly by the ROP method. The HBPG-PGA copolymers were semicrystalline with $T_{\mathrm{g}}=10-18^{\circ} \mathrm{C}$ and $T_{\mathrm{m}}$ in the range of $170-190^{\circ} \mathrm{C}$. The aggregation of HBPG-PLA in water leads to the formation of nanoparticles with the core-corona structure. The synthesis of HBPG-PLGA copolymers by the carbodiimide method was described. These copolymers could form nanoparticles functionalized with antibodies to transferrin [90]. In several studies, the ability to regulate the size and morphology of HBPGPLA-based nanostructures, to encapsulate therapeutic agents in them, and to release them in a controlled manner was revealed. It was shown that the hydrophobic shell of nanoparticles based on HBPG-PLA is capable of controlling the release rate of encapsulated water-soluble drugs [91-96]. To consider the possibility of using HBPG-like structures in neurology, T. Liu et al. developed a new drug delivery system to the brain based on HBPG-PLGA nanoparticles, the surface of which was functionalized with an antibody to transferrin. Nanoparticles were obtained by the double emulsion method [128].

\section{BRANCHED POLYMERS BASED ON BOLTORN POLYESTERS}

Owing to broad possibilities of modification and potential use in medicine, there are currently commercially available hyperbranched polymers: polyamidoamine (PAMAM) and polypropyleneimine (PPI), also known as Astromol, as well as Boltorn brand polyesters. One of the promising hyperbranched polymers is 
hyperbranched polyester obtained by the condensation of 2,2-bis(methylol) propionic acid with polyatomic alcohols (the trade name is Boltorn polyester). Entirely built from biocompatible, biodegradable blocks, this polyester can be used as a "platform" for the synthesis of a variety of compounds that can be used in a wide range of human activities, including targeted drug delivery. In this case, it is extremely important to study the structure of both the initial polyester and its modified derivatives. Boltorn is an aliphatic hyperbranched polyester synthesized by the polycondensation of 3-hydroxy-2-(hydroxymethyl)-2-methylpropionic acid (or 2,2-bis(methylol)propionic acid, bis(MPA)), which is a monomer of type $\mathrm{AB}_{2}$ and contains one carboxyl $(\mathrm{A}=-\mathrm{COOH})$ and two hydroxyl $(\mathrm{B}=-\mathrm{OH})$ functional groups. The synthesis of hyperbranched polymers based on bis(MPA) can be carried out using a hydroxyl-containing "core" $B_{f}(f$ is the number of functional hydroxyl groups) and without it. Boltorn polyes- ters of grades $\mathrm{H} 20, \mathrm{H} 30$, and $\mathrm{H} 40$ are progressively branching dendrite-like macromolecules containing three branches (in the case of trimethylolpropane as a branching center) with a large number of hydroxyl and ester groups. They are biocompatible biodegradable polymers and have bioavailability and low toxicity $\left(\mathrm{LD}_{50}=2000 \mathrm{mg} / \mathrm{kg}\right)$. Polyester polyols of this series are slightly soluble in water, and with an increase in the generation number, their solubility in water decreases. Their hydrophobicity is less pronounced than the hydrophobicity of polyolefins. It is known that, with an increase in the generation number of hyperbranched polymers, their degree of branching increases, as well as the size and capacity for guest molecules.

In the structure of polyester Boltorn, in addition to the branching center (core), there are linear, dendritic, and terminal units, from the ratio of which the degree of branching can be calculated:

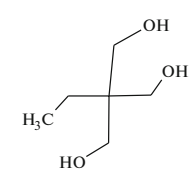

Trimethylolpropane

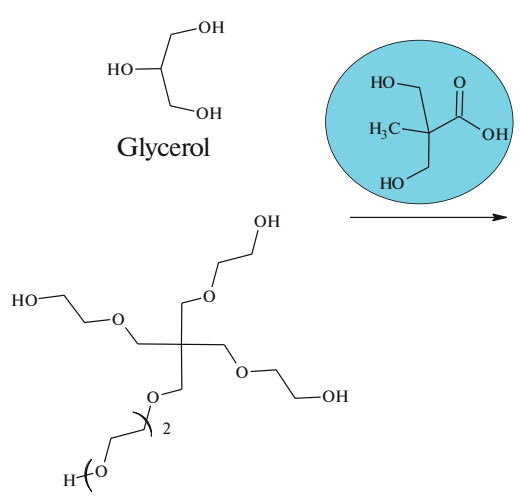

Ethoxylated pentaerythritol

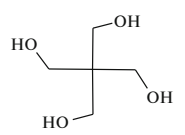

Pentaerythritol

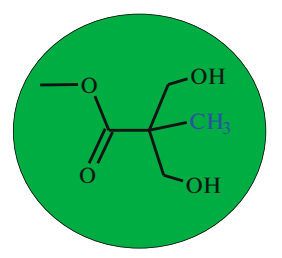

Terminal

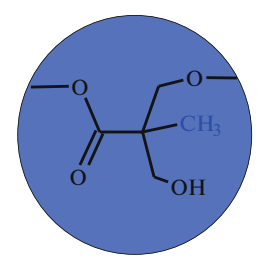

Linear

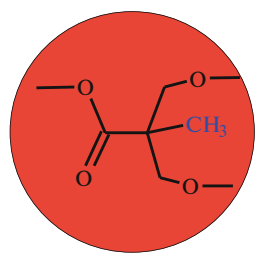

Dendritic 
Table 2. Boltorn-PLA hyperbranched polymers (catalyst $\mathrm{Sn}(\mathrm{Oct})_{2}$ )

\begin{tabular}{|c|c|c|c|c|}
\hline Core structure & Arm structure & Number of PLA arms & $\begin{array}{c}\text { Molecular weight } \\
\text { of copolymer } M_{\mathrm{n}} \times 10^{-3}\end{array}$ & References \\
\hline Boltorn H20 & PLLA & 16 & $17.8-157.7$ & [115] \\
\hline Boltorn H20 & PLLA- $b$-PCL & 16 & $51.8-104.3$ & [114] \\
\hline Boltorn H20 & $\begin{array}{l}\text { PLLA } \\
\text { PLLA- } b-\text { PTMC }\end{array}$ & 16 & $\begin{array}{c}45.4 \\
45.4-56.0\end{array}$ & {$[116]$} \\
\hline Boltorn H40 & PDLA & 64 & 260.0 & [114] \\
\hline Boltorn H40 & $\begin{array}{l}\text { PLLA } \\
\text { PLLA- } b-T P G S\end{array}$ & 64 & $\begin{array}{l}26.0 \\
55.0\end{array}$ & {$[114]$} \\
\hline Boltorn H40 & PLLA- $b-$ PEG & 64 & 108.5 & [117] \\
\hline Boltorn H40 & $\begin{array}{l}\text { PLLA- } b-m \text { PEG } \\
\text { PLLA- } b-\text { PEG-FA }\end{array}$ & 20 & 108.5 & [118] \\
\hline Boltorn H40 & PDLLA & 26 & 26.0 & {$[114]$} \\
\hline
\end{tabular}

The active sites are hydroxyl groups which are located in terminal and linear units. The presence of a large number of free hydroxyl groups (both inside and around the periphery of macromolecules) makes them especially attractive for chemical modification for use in biology and medicine. As a result of this modification, hyperbranched macromolecules were obtained containing carboxyl, amino, amido, and silylamine groups along the periphery as well as sulfur, phosphorus, and fluorine-containing groups. The modified polymers acquire the ability to self-assemble in aqueous solutions and to form nanostructures capable of retaining drugs. It was shown that PEG-modified Boltorn polyesters show promise for the controlled release of doxorubicin in breast cancer therapy [97, 98].

In a number of works, to increase the circulation time and drug loading, Boltorn $\mathrm{H} 40$ was modified with poly $(L$-lactide) [99], poly( $\varepsilon$-caprolactone) [100-102], poly $(L$-aspartate) [103], and poly ( $L$-glutamate) [104], after which they were hydrophilized with PEG blocks to formulate nanostructures.

Multiarm linear dendritic polymers based on Boltorn, commonly, are synthesized by the ROP of lactones in bulk in an inert medium at temperatures of $140-160^{\circ} \mathrm{C}$. The terminal hydroxyl groups are modified either with PEG, which makes it possible to increase the circulation time in vivo, or with folates or RGD peptides [105-113]. Table 2 provides a list of linear dendritic copolymers described in the literature.

In linear dendritic block copolymers BoltornPLA-PEG, polylactide blocks facilitate drug loading, as is evident, for example, when doxorubicin is covalently attached to them through $\mathrm{pH}$-sensitive bonds $[110,119]$. This advantage is perhaps less apparent in the case of physical drug loading, since hydrophobic compounds, such as paclitaxel, can also be physically loaded in pegylated Boltorn $\mathrm{H} 40$ without intermediate chains [120]. Boltorn H40 was used to develop aminofunctionalized systems as cationic carriers for nucleic acids [121], similar to what was studied using hyperbranched poly(amino esters) [122, 123]. Compared to one of the best transfection agents based on hyperbranched poly(ethyleneimine), the amino-functional Boltorn H40 is generally less effective but is significantly less cytotoxic. For example, it was used at concentrations up to $1 \mathrm{mg} / \mathrm{mL}$ without any significant loss of cell viability [121]. For Boltorn derivatives, very low values of the critical aggregation concentration in the range of several $\mu \mathrm{g} / \mathrm{mL}$ are usually observed [101, 102, $110,124]$, and they may simply reflect the insufficient sensitivity of analytical methods (usually determined by pyrene fluorescence) than the actual disaggregation, which would confirm the concept of hydrophobic domains with a very little concentration dependence. In addition, these systems usually range in size from several tens of nanometers to $100-200 \mathrm{~nm}$, which, nevertheless, suggests the multimolecular nature of colloidal particles. Monomolecular micellar nanosized preparations based on Boltorn-PCL modified with PEG and malic acid were obtained [125]. Some PEG blocks were modified with folic acid, and DOX was conjugated through $\mathrm{pH}$-sensitive hydrazone bonds which were cleaved in an acidic medium [125]. Similarly, octreotide was conjugated to BoltornPLLA-PEG [126, 127] and demonstrated an increased anticancer activity.

\section{CONCLUSIONS}

The last decade has witnessed a marked rise of interest in biodegradable polyesters for use in medicine and pharmaceuticals. The review shows that currently methods have been developed for the synthesis of hydrophobic and amphiphilic biodegradable polymers of various topologies: linear, star-shaped, and hyperbranched. Such a wide range of structures and formulations allow the flexible design of drug delivery systems, choosing the optimal material for each active agent. The development of new catalysts as well as 
methods for the synthesis of functional biodegradable polymers with controlled topology and physicochemical characteristics is an urgent area of research.

\section{FUNDING}

The work was partially supported by the Russian Science Foundation (project 18-73-10079 dated August 1, 2018). The sections on PLGA polymers and polyglycerol-based materials were partially supported by the National Research Center Kurchatov Institute (grant 1058 dated July 2, 2020).

\section{OPEN ACCESS}

This article is licensed under a Creative Commons Attribution 4.0 International License, which permits use, sharing, adaptation, distribution and reproduction in any medium or format, as long as you give appropriate credit to the original author(s) and the source, provide a link to the Creative Commons license, and indicate if changes were made. The images or other third party material in this article are included in the article's Creative Commons license, unless indicated otherwise in a credit line to the material. If material is not included in the article's Creative Commons license and your intended use is not permitted by statutory regulation or exceeds the permitted use, you will need to obtain permission directly from the copyright holder. To view a copy of this license, visit http://creativecommons.org/licenses/by/4.0/.

\section{REFERENCES}

1. T. S. Demina, A. B. Gilman, and A. N. Zelenetskii, High Energy Chem. 51, 302 (2017).

2. L. S. Nair and C. T. Laurencin, Prog. Polym. Sci. 32, 762 (2007).

3. T. S. Demina, Yu. S. Sotnikova, A. V. Istomin, Ch. Grandfils, T. A. Akopova, and A. N. Zelenetskii, Adv. Mater. Sci. Eng. 2018, article ID 8518016 (2018).

4. C. Jerome and P. Lecomte, Adv. Drug Delivery Rev. 60, 1056 (2008).

5. T. S. Demina, T. A. Akopova, A. N. Zelenetskii, L. V. Vladimirov, E. A. Markvicheva, and C. Grandfils, Mater. Sci. Eng., C 59, 333 (2016).

6. K. T. Kalinin, N. G. Sedush, P. V. Dmitryakov, and S. N. Chvalun, Chem. Open 9, 1027 (2020).

7. R. H. Platel, L. M. Hodgson, and C. K. Williams, Polym. Rev 48, 11 (2008).

8. D. J. Sawyer, Macromol. Symp. 201, 271 (2003).

9. K. V. Malafeev, O. A. Moskalyuk, V. E. Yudin, N. G. Sedush, S. N. Chvalun, V. Yu. Elokhovskii, E. N. Popova, and E. M. Ivan'kova, Polym. Sci., Ser. A 59, 53 (2017).

10. I. A. Shuklov, H. Jiao, A. Börner, J. Schulze, W. Tietz, and K. Kühlein, Tetrahedron Lett. 52, 1027 (2011).

11. V. I. Gomzyak, V. A. Demina, E. V. Razuvaeva, N. G. Sedush, and S. N. Chvalun, Fine Chem. Technol. 12 (5), 5 (2017).

12. S. Penczek, M. Cypryk, A. Duda, P. Kubisa, and S. Slomkowski, Prog. Polym. Sci. 32, 247 (2007).
13. P. Gunatillake, R. Mayadunne, and R. Adhikari, Biotechnol. Annu. Rev. 12, 301 (2006).

14. J. Pradip, S. Mousumi, S. Sneha, J. Venkatesan, and D. Abhimanyu, Eur. Polym. J. 142, 110155 (2021).

15. J. C. Middleton and A. J. Tipton, Biomaterials 21, 2335 (2001).

16. T.-W. Chung, Y.-Y. Huang, and Y.-Z. Liu, Int. J. Pharm. 212, 161 (2001).

17. A. J. Almeida, H. Alpar, and M. Brown, J. Pharm. Pharmacol. 45, 198 (1993).

18. R. Asmatulu, M. A. Zalich, R. O. Claus, and J. S. Riffle, J. Magn. Magn. Mater. 92, 108 (2005).

19. S. W. Zielhuis, J. F. W. Nijsen, R. Figueiredo, B. Feddes, A. M. Vredenberg, A. D. Van Het Schip, and W. E. Hennink, Biomaterials 26, 925 (2005).

20. M. Li, O. Rouaud, and D. Poncelet, Int. J. Pharm. 363, 26 (2008).

21. R. Arshady, J. Controlled Release 17, 1 (1991).

22. W. Chaisri, W. E. Hennink, and S. Okonogi, Curr. Drug Delivery 6, 69 (2009).

23. S. Mao, J. Xu, C. Cai, O. Germershaus, A. Schaper, and T. Kissel, Int. J. Pharm. 334, 137 (2007).

24. P. He, S. Davis, and L. Illum, Int. J. Pharm. 187, 53 (1999).

25. A. Perez, R. Hernández, D. Velasco, D. Voicu, and C. Mijangos, J. Colloid Interface Sci. 441, 90 (2015).

26. Q. Xu, M. Hashimoto, T. Dang Tram, T. Hoare, D. S. Kohane, G. M. Whitesides, R. Langer, and D. G. Anderson, Small 5, 1575 (2009).

27. E. A. Petrova, S. A. Kedik, K. V. Alekseev, E. V. Blynskaya, A. V. Panov, V. V. Suslov, and N. V. Tikhonova, Pharm. Chem. J. 48, 65 (2014).

28. H. K. Makadia and S. J. Siegel, Polymers 3, 1377 (2011).

29. L. Y. Lee, S. H. Ranganath, Y. Fu, J. L. Zheng, H. S. Lee, C.-H. Wang, and K. A. Smith, Chem. Eng. Sci. 64, 4341 (2009).

30. M. Alonso-Sande, A. des Rieux, V. Fievez, B. Sarmento, A. Delgado, C. Evora, C. Remuñán-López, V. Préat, and M. J. Alonso, Biomacromolecules 14, 4046 (2013).

31. Y. N. Konan, C. Radovan, F. Joselyne, B. Myriam, R. Gurny, and E. Allémann, Eur. J. Pharm. Biopharm. 55, 115 (2003).

32. S. J. Siegel, J. B. Kahn, K. Metzger, K. I. Winey, K. Werner, and N. Dan, Eur. J. Pharm. Biopharm. 64, 287 (2006).

33. P. Goddard, E. Hutchinson Lusie, J. Brown, and L. J. Brookman, J. Controlled Release 10, 5 (1989).

34. K. J. Zhu, L. Xiangzhou, and Y. Shilin, J. Appl. Polym. Sci. 39, 1 (1990).

35. H. Asadi, K. Rostamizadeh, D. Salari, and M. Hamadi, Int. J. Pharm. 416, 356 (2011).

36. J. L. Hedrick, M. K. Kiesewetter, E. J. Shin, and R. M. Waymouth, Macromolecules 43, 2093 (2010).

37. M. Vittaz, D. Bazile, G. Spenlehauer, T. Verrecchia, M. Veillard, F. Puisieux, and D. Labarre, Biomaterials 17, 1575 (1996).

38. D. Lee, I. Teraoka, T. Fujiwara, and Y. Kimura, J. Chromatogr. A 966, 41 (2002). 
39. C. Bouissou, J. J. Rouse, R. Price, and C. F. van der Walle, Pharm. Res 23, 1295 (2006).

40. S. Angot, K. S. Murthy, D. Taton, and Y. Gnanou, Macromolecules 31, 7218 (1998).

41. W. Yuan, L. Zhu, X. Huang, S. Zheng, and X. Tang, Polym. Degrad. Stab. 87 (3), 503 (2005).

42. M. Li, N. M. Jahed, K. Min, and K. Matyjaszewski, Macromolecules 37, 2434 (2004).

43. K. Matyjaszewski, P. J. Miller, J. Pyun, G. Kickelbick, and S. Diamanti, Macromolecules 32, 6526 (1999).

44. K. Matyjaszewski and S. G. Gaynor, Macromolecules 30, 7042 (1997).

45. H. Gao and K. Matyjaszewski, Prog. Polym. Sci. 34, 317 (2009).

46. H. Gao and K. Matyjaszewski, Macromolecules 39, 3154 (2006).

47. H. Gao and K. Matyjaszewski, Macromolecules 40, 399 (2007).

48. C.-M. Dong, K.-Y. Qiu, Z.-W. Gu, and X.-D. Feng, Macromolecules 34, 4691 (2001).

49. E. A. Rainbolt, K. E. Washington, M. C. Biewer, and M. C. Stefan, Polym. Chem. 62369 (2015).

50. W. Zhang and S. Zheng, Polym. Bull. 58, 767 (2007).

51. M. Yuan, Z. He, H. Li, and L. Jiang, Polym. Bull. 71, 1331 (2014).

52. S. Matsumura, K. Mabuchi, and K. Toshima, Macromol. Rapid Commun. 18, 477 (1997).

53. A. Michalski, T. Makowski, T. Biedron, M. Brzezinski, and T. Biela, Polymer 90, 242 (2016).

54. Z. Li, B. H. Tan, T. Lin, and C. He, Prog. Polym. Sci. 62, 22 (2016).

55. K. Numata, R. K. Srivastava, A. Finne-Wistrand, A. C. Albertsson, Y. Doi, and H. Abe, Biomacromolecules 8, 3115 (2007).

56. Q. Hao, F. Li, Q. Li, Y. Li, L. Jia, J. Yang, Q. Fang, and A. Cao, Biomacromolecules 6, 2236 (2005).

57. I. Arvanitoyannis, A. Nakayama, E. Psomiadou, N. Kawasaki, and N. Yamamoto, Polymer 37, 651 (1996).

58. A. Kundys, A. Plichta, Z. Florjanczyk, A. Zychewicz, P. Lisowska, P. Parzuchowski, and E. Wawrzynska, Polym. Int. 65, 927 (2016).

59. H. R. Kricheldorf, H. Hachmann-Thiessen, and G. Schwarz, Biomacromolecules 5, 492 (2004).

60. H. Tsuji, T. Miyase, Y. Tezuka, and S. K. Saha, Biomacromolecules 6, 244 (2005).

61. M. Socka, M. Brzezinski, A. Michalski, A. Kacprzak, T. Makowski, and A. Duda, Langmuir 34, 3701 (2018).

62. L. Wang and C. M. Dong, J. Polym. Sci., Part A: Polym. Chem. 44, 2226 (2006).

63. S. H. Kim, Y. K. Han, Y. H. Kim, and S. I. Hong, Makromol. Chem. 193, 1623 (1992).

64. C. Cai, L. Wang, and C. Dong, J. Polym. Sci., Part A: Polym. Chem. 44, 2034 (2006).

65. M. P. Shaver and D. Cameron, Biomacromolecules 11, 3673 (2010).

66. M. Xie, J. Ge, Y. Xue, Y. Du, B. Lei, and P. X. Ma, J. Mech. Behav. Biomed. Mater. 51, 163 (2015).
67. H. Asadi, K. Rostamizadeh, D. Salari, and M. Hamadi, Int. J. Pharm. 416, 356 (2011).

68. C. A. P. Joziasse, H. Grablowitz, and A. J. Pennings, Macromol. Chem. Phys. 201, 107 (2000).

69. B. Atthoff, M. Trollsås, H. Claesson, and J. L. Hedrick, Macromol. Chem. Phys. 200, 1333 (1999).

70. O. Coulembier, M. K. Kiesewetter, A. Mason, P. Dubois, J. L. Hedrick, and R. M. Waymouth, Angew. Chem., Int. Ed. 46, 4719 (2007).

71. S.-H. Luo, Q.-F. Wang, J.-F. Xiong, and Z.-Y. Wang, J. Polym. Res. 19, 9962 (2012).

72. B. S. Lele and J. C. Leroux, Polymer 43, 5595 (2002).

73. S. H. Kim and Y. H. Kim, Macromol. Symp. 144, 277.

74. Y. Lin, A. Zhang, and L. Wang, J. Appl. Polym. Sci. 124, 4496 (2012).

75. A. R. Goddard, S. Pérez-Nieto, S. M. Howdle, K. Carmichael, T. M. Passos, B. Quilty, and D. J. Irvine, Green Chem. 18, 4772 (2016).

76. X.-H. Dai, Z.-M. Wang, W. Liu, C.-M. Dong, J.-M. Pan, S.-S. Yuan, Y.-S. Yan, D.-M. Liu, and L. Sun, Colloid Polym. Sci. 292, 2111 (2014).

77. Y. Zhao, X. Shuai, C. Chen, and X. Fu, Macromolecules 37, 8854 (2004).

78. I. Arvanitoyannis, A. Nakayama, N. Kawasaki, and N. Yamamoto, Polymer 36, 2947 (1995).

79. E. Doganci, M. A. Tasdelen, and F. Yilmaz, Macromol. Chem. Phys. 216, 1823 (2015).

80. X. H. Dai and C. M. Dong, J. Polym. Sci., Part A: Polym. Chem. 46, 817 (2008).

81. P.-F. Gou, W.-P. Zhu, and Z.-Q. Shen, Biomacromolecules 11, 934 (2010).

82. F. Wang, R. D. Rauh, T. K. Bronich, A. V. Kabanov, and J. Roovers, Bioconjugate Chem. 19, 1423 (2008).

83. B. I. Voit, J. Polym. Sci., Part A: Polym. Chem. 38, 2505 (2000).

84. M. Jikei and M. Kakimoto, J. Polym. Sci., Part A: Polym. Chem. 42, 1293 (2004).

85. M. P. A. Lim, W. L. Lee, E. Widjaja, S. Chye, and J. Loo, Biomater. Sci. 1, 486 (2013).

86. A. Burgath, A. Sunder, I. Neuner, R. Mülhaupt, and H. Frey, Macromol. Chem. Phys. 201, 792 (2000).

87. C. Gottschalk, F. Wolf, and H. Frey, Macromol. Chem. Phys. 208, 1657 (2007).

88. X. Gao, X. Zhang, X. Zhang, Y. Wang, L. Sun, and C. Li, J. Pharm. Pharmacol. 63, 757 (2011).

89. F. K. Wolf, A. M. Fischer, and H. Frey, Beilstein J. Org. Chem. 6, 67 (2010).

90. M. C. Mohiuddin, A. Quadir, Q. Sunil, K. Sharma, and R. Haag, Adv. Mater. 22, 190 (2010).

91. Y. Deng, J. K. Saucier-Sawyer, C. J. Hoimes, J. Zhang, Y.-E. Seo, J. W. Andrejecsk, and W. M. Saltzman, Biomaterials 35, 6595 (2014).

92. M. R. Vijayakumar, M. S. Muthu, and S. Singh, Expert Opin. Drug Delivery 10, 529 (2013).

93. S. F. Mehrabadi, O. Hirsch, R. Zeisig, P. Posocco, E. Laurini, S. Pricl, R. Haag, W. Kemmner, and M. Calderón, RSC Adv. 5, 78760 (2015).

94. X. Gao, X. Zhang, Z. Wu, X. Zhang, and C. Li, J. Controlled Release 140, 141 (2009). 
95. S. Hecht, N. Vladimirov, and J. M. J. Fréchet, J. Am. Chem. Soc. 123, 18 (2001).

96. T. Biela, A. Duda, K. Rode, and H. Pasch, Polymer 44, 1851 (2003).

97. X. Zeng, R. Morgenstern, and A. M. Nystrom, Biomaterials 35, 1227 (2014).

98. J. Bao, X. Chang, Q. Xie, C. Yu, G. Shan, Y. Bao, and P. Pan, Macromolecules 50, 8619 (2017).

99. S. Chen, X.-Z. Zhang, S.-X. Cheng, R.-X. Zhuo, and W.-G. Zhong, Biomacromolecules 9, 2578 (2008).

100. S. Aryal, M. Prabaharan, S. Pilla, and S. Gong, Int. J. Biol. Macromol. 44, 346 (2009).

101. D. Wang, H. Chen, Y. Su, F. Qiu, L. Zhu, X. Huan, B. Zhu, D. Yan, F. Guo, and X. Zhu, Polym. Chem. 4, 85 (2013).

102. M. Prabaharan, J. J. Grailer, S. Pilla, D. A. Steeber, and S. Gong, Biomaterials 30, 5757 (2009).

103. Y. Xiao, H. Hong, A. Javadi, J. W. Engle, W. Xu, Y. Yang, Y. Zhang, T. Barnhart, W. Cai, and S. Gong, Biomaterials 33, 3071 (2012).

104. S. Corneillie and M. Smet, Polym. Chem. 6, 850 (2014).

105. M. C. Lukowiak, B. N. S. Thota, and R. Haag, Biotechnol. Adv. 33, 1327 (2015).

106. M. Prabaharan, J. J. Grailer, S. Pilla, D. A. Steeber, and S. Gong, Biomaterials 30, 3009 (2010).

107. H. Yang, X. Zhao, X. Zhang, L. Ma, B. Wang, and H. Wei, J. Polym. Sci., Part A: Polym. Chem. 56, 1383 (2018).

108. V. V. Istratov, T. V. Krupina, V. A. Vasnev, and V. I. Gomzyak, High Perform. Polym. 29, 708 (2017).

109. C. Sun, R. Sze, and M. Zhang, J. Biomed. Mater. Res., Part A 78, 550 (2006).

110. V. V. Istratov, V. I. Gomzyak, T. V. Krupina, V. A. Vasnev, and S. N. Chvalun, Polym. Sci., Ser. B 59, 730 (2017).

111. X. Li, Y. Qian, T. Liu, X. Hu, G. Zhang, Y. You, and S. Liu, Biomaterials 32, 6595 (2011).

112. Y. Wu, G. Xu, X. Jin, and X. Zhu, Sci. China Mater. 61 (11), 144 (2018).
113. S. J. T. Rezaei, H. S. Abandansari, M. R. Nabid, and H. Niknejad, J. Colloid Interface Sci. 425, 27 (2014).

114. W. Zhang and S. Zheng, Polym. Bull. 58, 767 (2007).

115. D. J. Coady, A. C. Engler, Y. Y. Yang, and J. L. Hedrick, Polym. Chem. 2, 2619 (2011).

116. K. S. Suneel, D. M. A. Buzza, D. J. Groves, T. C. B. McLeish, D. Parker, A. J. Keeney, and W. J. Feast, Macromolecules 35, 9605 (2002).

117. Smart Polymer Materials for Biomedical Applications, Ed. by S. Li, A. Tiwari, M. Prabaharan, and S. Aryal (Nova Sci. Publ., New York, 2010).

118. X. Yang, J. J. Grailer, S. Pilla, D. A. Steeber, and S. Gong, Bioconjugate Chem. 21, 496 (2010).

119. S. A. Kedik, E. S. Zhavoronok, I. P. Sedishev, A. V. Panov, V. V. Suslov, E. A. Petrova, M. D. Sapelnikov, D. O. Shatalov, and D. V. Eremin, Dev. Regist. Pharm. Prod. 2 (3), 18 (2013).

120. H. Chen, G. Li, H. Chi, D. Wang, C. Tu, L. Pan, L. Zhu, F. Qiu, F. Guo, and X. Zhu, Bioconjugate Chem. 23, 1915 (2012).

121. Y. Pang, Q. Zhu, J. Liu, J. Wu, R. Wang, S. Chen, X. Zhu, D. Yan, W. Huang, and B. Zhu, Biomacromolecules 11, 575 (2010).

122. D. Wu, Y. Liu, X. Jiang, C. He, S. H. Goh, and K. W. Leong, Biomacromolecules 7, 1879 (2006).

123. M. Ornatska, S. Peleshanko, K. L. Genson, B. Rybak, K. N. Bergman, and V. V. Tsukruk, J. Am. Chem. Soc. 126, 9675 (2004).

124. G. Ma, C. Zhang, L. Zhang, H. Sun, C. Song, C. Wang, and D. Kong, J. Mater. Sci.: Mater. Med. 27, 17 (2016).

125. S. Indoria, V. Singh, and M.-F. Hsieh, Int. J. Pharm. 582, 119314 (2020).

126. R. Jaskula-Sztul, W. Xu, G. Chen, A. Harrison, A. Dammalapati, R. Nair, Y. Cheng, S. Gong, and H. Chen, Biomaterials 91, 1 (2016).

127. X. Zeng, Y. Zhang, Z. Wu, P. Lundberg, M. Malkoch, and A. M. Nystrom, J. Polym. Sci., Part A: Polym. Chem. 50, 280 (2012).

128. H. Bao, X. Jin, L. Li, F. Lv, and T. Liu, J. Mater. Sci.: Mater. Med 23, 1891 (2012). 